EPJ Web of Conferences 47, 07001 (2013)

DOI: $10.1051 /$ epjconf/20134707001

(C) Owned by the authors, published by EDP Sciences, 2013

\title{
Lunar eclipses: Probing the atmosphere of an inhabited planet
}

\author{
A. García Muñoz
}

ESA/ESTEC, PB 299, 2200AG Noordwijk, The Netherlands

\begin{abstract}
The Moon's brightness during a lunar eclipse is indicative of the composition, cloudiness and aerosol loading of the Earth's atmosphere. The idea of using lunar eclipse observations to characterize the Earth's atmosphere is not new, but the interest raised by the prospects of discovering Earth-like exoplanets transiting their host stars has brought renewed attention to the method. We review some recent efforts made in the prediction and interpretation of lunar eclipses. We also comment on the contribution of the lunar eclipse theory to the refractive theory of planetary transits.
\end{abstract}

\section{INTRODUCTION}

Finding life outside our planet remains as one of humankind's unfilfilled major quests. To date, though, Earth is the only planet known to host life, which makes of it a sort of reference to which all other candidates are to be compared. Conditions for life are partly sustained by the Earth's climate system, that participates in the deposition of solar energy on the planet. Amongst the factors that control the climate, atmospheric aerosols affect the fraction of incident solar energy that is actually deposited. In the context of a remote observer attempting to characterize the Earth's spectral appearance, aerosols may additionally mask the spectroscopic signatures of atmospheric gases such as $\mathrm{O}_{2}, \mathrm{O}_{3}, \mathrm{CO}_{2}$ or $\mathrm{CH}_{4}$ often associated with life. These questions highlight the role of aerosols in the habitability and prospective characterization of Earth-like exoplanets.

Lunar eclipses occur when the Moon enters the Earth's shadow. During the transit, the appearance of the shadowed Moon varies notably as a result of the refraction, scattering and absorption of the solar light passing through the Earth's atmosphere. The same phenomenon of a lunar eclipse appears as an occultation to an observer sitting on the Moon. During the eclipse, the selenite observer would see the refracted image of the Sun as a luminous halo spanning part or the entire of the Earth's terminator. Being readily accessible to naked-eyed observers, there have been written reports of lunar eclipses over centuries.

The appearance of the shadowed Moon varies from eclipse to eclipse, a fact that is directly connected with the prevalent aerosol conditions in the atmosphere at the time of the event. Phenomena such as volcanic eruptions or meteor showers, both of which alter to different extents the concentration of stratospheric particles, have often been proposed to explain unusually dark eclipses. Keen [8] gave clear evidence that the largest volcanic eruptions between 1960 and 1982 were followed by total eclipses that appeared exceptionally dark. Over that period, the Agung and El Chichón eruptions, in 1963 and 1982, respectively, resulted in particularly dark eclipses. Eruptions of Volcanic Explosivity Index $\geq 3$ have the capacity of injecting significant amounts of ash and $\mathrm{SO}_{2}$ into the upper troposphere and lower

\footnotetext{
ae-mail: agarcia@rssd.esa.int; tonhingm@gmail.com
}

This is an Open Access article distributed under the terms of the Creative Commons Attribution License 2.0, which permits unrestricted use, distribution, and reproduction in any medium, provided the original work is properly cited. 
stratosphere. Once in the stratosphere, the $\mathrm{SO}_{2}$ turns into $\mathrm{H}_{2} \mathrm{SO}_{4}$ droplets that are carried over by highaltitude winds, thus contributing to the global aerosol loading of the planet. The redistribution of aerosols over planetary scales may take weeks or months. Once they reach the stratosphere, the lifetime of $\mathrm{H}_{2} \mathrm{SO}_{4}$ droplets may be of months or years [1], which means that eclipses up to a few years after a large volcanic eruption may appear affected. Additionally, it has recently been noted [4] that the ash and burning biomass injected into the stratosphere by large wildfires [2] might also have a local impact on the darkening of the eclipsed Moon.

\section{THE LUNAR ECLIPSE THEORY}

The quantitative interpretation of the Moon's appearance calls for a rigorous theoretical formulation. Link summarized in a number of works much of the knowledge relevant to lunar eclipses and other astronomical phenomena in which refraction is essential $[9,10]$. The residual brightness of the shadowed Moon contains the contributions of direct and scattered sunlight. The direct contribution is associated with photons that traverse the entire Earth's limb without undergoing scattering collisions with the medium and following paths curved by the atmosphere's vertical gradient of refractivity. The scattered component is made up of photons that undergo one or more scattering collisions with the gas molecules and aerosols in the atmosphere before reaching the Moon.

The lunar eclipse theory has recently been revisited [3]. The effort was motivated by modern computational capacities, which allow one to solve the Radiative Transport Equation (RTE) in a relatively straightforward manner. Also, the progress made over the last decades in the understanding of tropospheric/stratospheric aerosols justifies a new look at the subject.

In the formulation of Ref. [3], the classical RTE:

$$
\mathbf{s} \cdot \nabla L(\mathbf{x}, \mathbf{s})=-\gamma(\mathbf{x}) L(\mathbf{x}, \mathbf{s})+\beta(\mathbf{x}) \int_{\partial \Omega} p\left(\mathbf{x}, \mathbf{s}, \mathbf{s}^{\prime}\right) L\left(\mathbf{x}, \mathbf{s}^{\prime}\right) d \Omega\left(\mathbf{s}^{\prime}\right),
$$

is solved at the observer's site on the lunar surface. Here, $L(\mathbf{x}, \mathbf{s})$ is the radiance at $\mathbf{x}$, and $\mathbf{s}$ and $\mathbf{s}^{\prime}$ are direction vectors. $\gamma(\mathbf{x})$ and $\beta(\mathbf{x})$ are the extinction and scattering coefficients, respectively, and $p\left(\mathbf{x}, \mathbf{s}, \mathbf{s}^{\prime}\right) d \Omega\left(\mathbf{s}^{\prime}\right)$ is the scattering phase function over the differential solid angle $d \Omega\left(\mathbf{s}^{\prime}\right)$.

For the direct sunlight component, the RTE is complemented with Snell's law for the refraction of light in a spherically-symmetric medium. The direct radiance is integrated over the solid angle that forms the refracted image of the Sun at the Earth's terminator. The image is determined by ray tracing from the observer's site back to the solar disk after solving the system of ordinary differential equations for the ray paths. Limb darkening of the solar emission is optionally taken into consideration in the model. For the scattered sunlight component, a Monte Carlo scheme is utilized to integrate the photon trajectories over the Earth's terminator.

\section{THE EARTH OCCULTING THE SUN FOR A MOON-BOUND OBSERVER}

Figure (1; from Ref. [3]) shows model simulations of the refracted halo at the Earth's terminator as viewed from the Moon. Parameter $e$ is the geocentric angular distance of the observer's site to the umbra axis. The umbra/penumbra edge occurs at $e \sim 0.7^{\circ}$.

Two series of television images of the Earth occulting the Sun were obtained by the Surveyor III from the Moon's surface in April 1967 [15]. The images show in different color filters the refracted image of the Sun at the Earth's terminator. The refracted halo extends over both hemispheres. Interestingly, the halo shows clearly discontinuous structure revealing the occurrence of clouds in the solar path. A ground-based observer looking in the direction of Surveyor III would measure a brightness that is essentially the integrated signal of the refracted halo captured by the Surveyor III television images. 


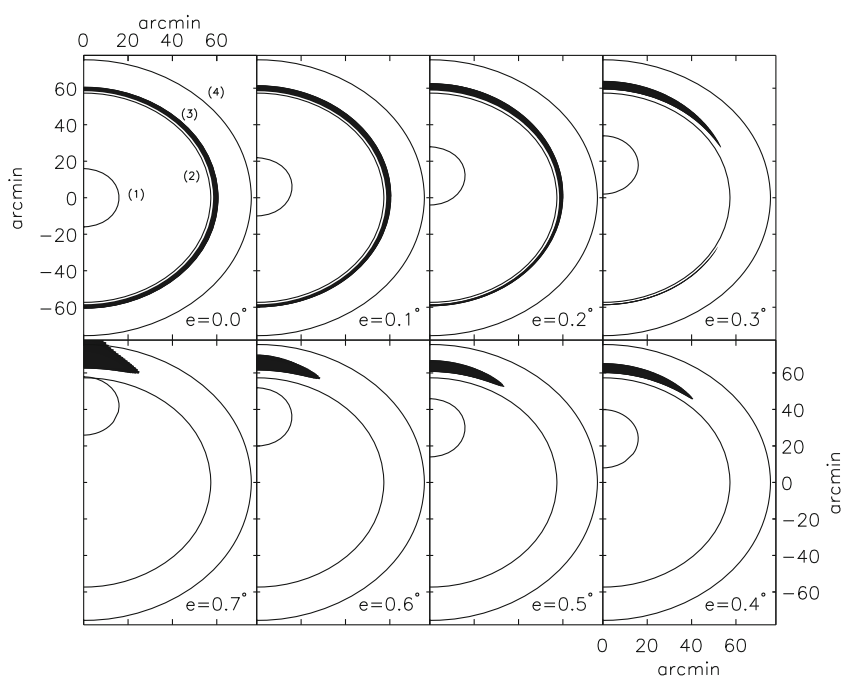

Figure 1. Refracted image of the Sun at the Earth's terminator for a number of umbra phases. From Ref. [3].

More recent imagery has been obtained by the Kaguya/Selene explorer [20] during the penumbral partial eclipse of February 2009. The Kaguya/Selene footage shows, in addition to the Sun rising from behind the Earth, (most of) the ring of scattered sunlight circling the Earth's disk.

\section{LUNAR ECLIPSE PHOTOMETRY}

There is a long record of photometric measurements of the umbra shadow cast upon the lunar disk. Generally, photometric observations helped establish the connection between the umbra darkness and atmospheric conditions. Spatially-resolved photometry provides access to the atmospheric conditions over a range of altitudes along the Earth's terminator. Then, the inversion of the measured intensities to yield vertically-resolved limb opacities is feasible.

Photometric measurements obtained with broadband filters are often used to infer properties of the atmospheric aerosol loading $[11,17]$. Narrowband filters are prefered to probe specific molecular features such as $\mathrm{H}_{2} \mathrm{O}$ [18]. Frequently, spatially-resolved measurements show that the darkest location in the umbra is offset from the umbra axis, a result from the obvious fact that the Earth's atmospheric composition and aerosol loading are not symmetric. In addition, photometric measurements give evidence of the wavelength-dependence of the optical properties of the atmospheric gas and aerosols. Typically, the drop in brightness towards the center of the umbra is faster at the shorter wavelengths. Longwards of about $4 \mu \mathrm{m}$, the light received from the Moon becomes predominantly thermal radiation from the lunar surface. Thermal radiation measurements of the eclipsed Moon have been utilized to investigate the thermal inertia of the lunar surface [14].

\section{LUNAR ECLIPSE SPECTROSCOPY}

Pallé et al. [12] published a spectrum of the umbra during the partial eclipse of 16 August 2008. The observations were made at two telescopes of the Observatorio del Roque de los Muchachos (La Palma, Spain). The spectrum from 0.4 to $1 \mu \mathrm{m}$ was obtained with the ALFOSC instrument at the Nordic Optical Telescope. Several spectra from 1 to $2.4 \mu \mathrm{m}$ were obtained with the LIRIS instrument at the William Herschel Telescope. The infrared spectra probed different phases of the eclipse or, equivalently, 


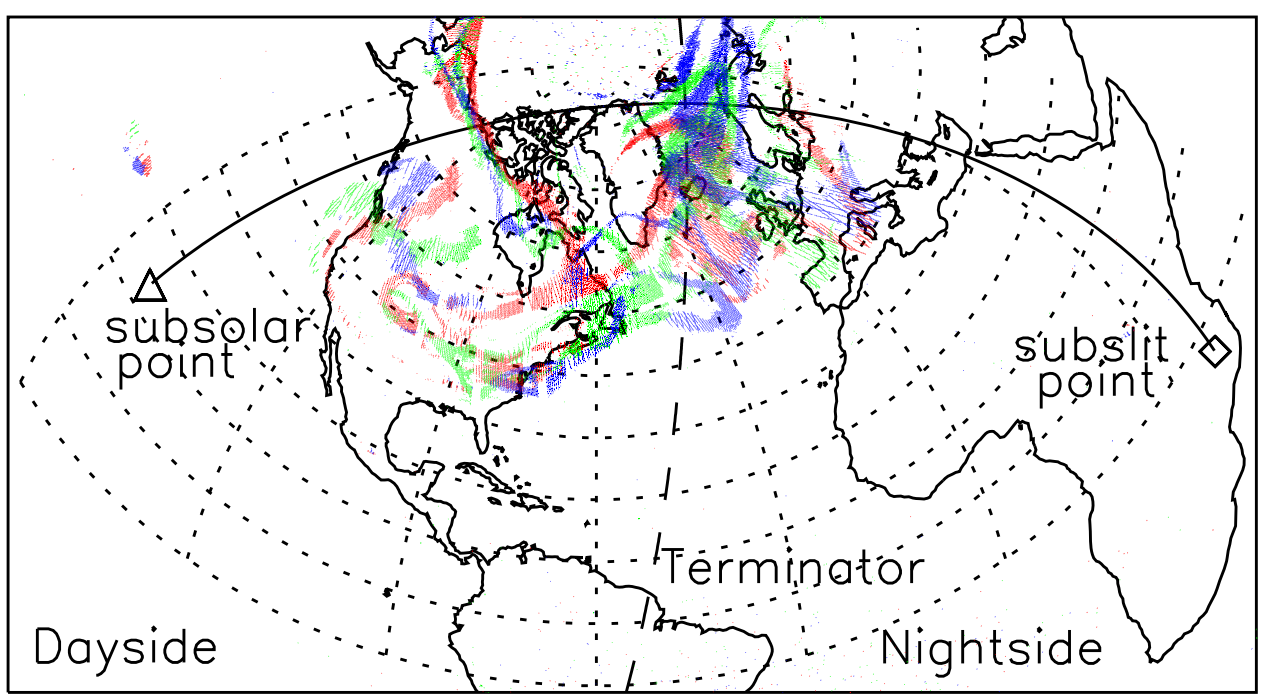

Figure 2. Mid-section path of the sunbeam that reached the lunar disk in umbra targeted by the Nordic Optical Telescope at 21:36UT. Overplotted, the $\mathrm{SO}_{2}$ cloud on 15, 16 and 17 August (red, green and blue, respectively) according to AURA/OMI data (downloaded from the Giovanni online data system, developed and maintained by the NASA GES DISC).

different separations from the umbra center. The spectrum published in Ref. [12] combines the ALFOSC spectrum and the three LIRIS spectra closest to the umbra center. It was claimed that the spectrum represented the Earth's transmission spectrum, i.e. the spectrum of Earth as observed from a remote distance while transiting the Sun.

The published spectrum shows clear evidence of molecules such as $\mathrm{O}_{2}, \mathrm{O}_{3}, \mathrm{H}_{2} \mathrm{O}, \mathrm{CO}_{2}$ and $\mathrm{CH}_{4}$. It also shows two prominent broad features at 1.06 and $1.27 \mu \mathrm{m}$ due to the Collision Induced Absorption bands of $\mathrm{O}_{2} \cdot \mathrm{O}_{2}$ and $\mathrm{O}_{2} \cdot \mathrm{N}_{2}$ [5], thus suggesting that the spectrum's structure is formed in the lowermost atmosphere near the surface.

Interestingly, the ALFOSC spectrum levels off shortwards of $600 \mathrm{~nm}$, a fact suggesting that it is dominated by scattered sunlight at the shorter wavelengths. García Muñoz et al. [4] were able to reproduce the ALFOSC data with a synthetic spectrum consisting of both refractive and scattered components. For a value $e=0.34^{\circ}$ of the angular distance to the umbra center, the authors produced a library of synthetic spectra with the ozone abundance and the extinction and Ångstrom coefficient for aerosols as free parameters. The best fit to the observations indicated elevated aerosol extinctions and an Ångstrom coefficient consistent with moderately large aerosols.

Further, Ref. [4] argues that the occurrence of a volcanic eruption on the Aleutian Islands, Alaska, about one week before the lunar eclipse plausibly provided the needed atmospheric extinction. Indeed, the three explosions on 7-8 August 2008 of the Kasatochi volcano terminated a period, started in 1997, characterized by low stratospheric aerosol amounts. That period had been preceded by 5-6 years of dilution of the volcanic matter injected by the 1991 eruption of Mt. Pinatubo. The Kasatochi eruption injected vast amounts of ash, $\mathrm{SO}_{2}$ and other gases into the stratosphere. The volcanic cloud progressed rapidly across North America and the Northern Atlantic, and reached the European continent about one week after the eruption. The progress of the volcanic cloud was monitored with unprecedented detail from satellites, aerial platforms and the ground. Figure (2) displays the $\mathrm{SO}_{2}$ column, an usual plume tracer, measured with AURA/OMI on 15-17 August 2008. The figure displays also the arc of the great circle that joins the subsolar point and the subslit point at the time of the ALFOSC observations. The arc traces the projected mid-section of the sunbeam that reaches the lunar disk targeted by the 


\section{Hot Planets and Cool Stars}

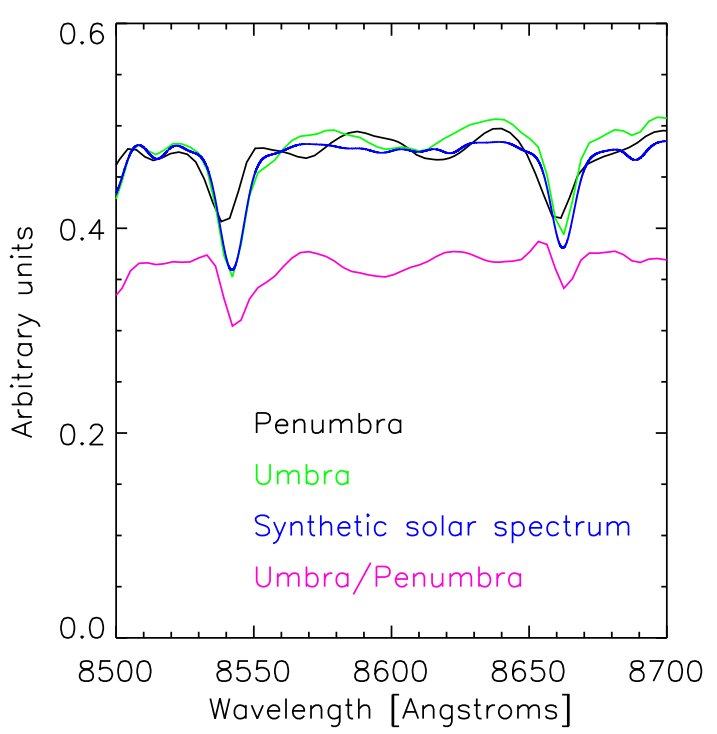

Figure 3. Spectral region near the NIR triplet of $\mathrm{Ca}^{+}$. See text for comments.

telescope. It is apparent that, before arriving at the Moon, the sunbeam crossed the bulk of the volcanic cloud over the North Atlantic Ocean.

García Muñoz et al. [7] investigated the data obtained with LIRIS at various phases of the eclipse for wavelengths longer than $1 \mu \mathrm{m}$. The spectra reveal that the depth of spectroscopic features varies with the distance to the umbra center. A direct comparison of the eclipse spectrum of Ref. [12] with SCIAMACHY/ENVISAT solar occultation data indicates that the eclipse spectrum forms preferentially at altitudes of closest approach of up to $10 \mathrm{~km}$. The conclusion is consistent with the ray tracing of the refracted solar image at the Earth's terminator [3].

The discussion of the altitudes being probed for $e=0.34^{\circ}$ is relevant to the interpretation of the atomic features identified in the eclipse spectrum of Ref. [12]. The initial assignment [12, 13] included features of CaII at 393.4 and $396.8 \mathrm{~nm}$ in the ultraviolet and 849.8, 854.2, 866.2 $\mathrm{nm}$ in the near infrared, as well as the $\mathrm{Na}$ doublet at 589 and $589.6 \mathrm{~nm}$. The evidence for $\mathrm{Ca}^{+}$in the UV and $\mathrm{Na}$ in the visible was subsequently reconsidered [4, 7]. In the NIR, the situation for $\mathrm{Ca}^{+}$is exposed with the aid of Fig. (3). The black and green curves are spectra of the light reflected from the Moon in umbra and penumbra, respectively. The black curve is a high-resolution synthetic spectrum of the Sun degraded to the ALFOSC resolving power. It is apparent that the umbra spectrum conforms to the shape of the solar spectrum, which suggests the absence of atmospheric $\mathrm{Ca}^{+}$absorption. For some reason, though, the penumbra spectrum differs notably (being less deep) from the synthetic solar spectrum (blue curve) at the line cores. The umbra/penumbra ratio of spectra (purple curve; the so-called lunar eclipse spectrum of Ref. [12]) shows dips at the $\mathrm{Ca}^{+}$solar line positions that reflect (in part, at least) the mismatch between the observed penumbra spectrum and the synthetic solar spectrum. It is challenging to explain the reason for the mismatch between the penumbra and synthetic solar spectrum.

Vidal-Madjar et al. [19] published a spectrum of the Moon in penumbra from 400 to $700 \mathrm{~nm}$ for, also, the 16 August 2008 eclipse. Their spectrum shows evidence of $\mathrm{O}_{2}, \mathrm{O}_{3}$ and, with more difficulties, $\mathrm{Na}$. The detectability of the latter is facilitated by the effectively higher altitudes of the atmosphere probed in a penumbra observation and the high resolution of their spectrum. 


\section{EPJ Web of Conferences}

\section{LUNAR ECLIPSES AND EXOPLANETARY TRANSITS}

Refraction is a key phenomenon in the prediction and interpretation of lunar eclipses. Thus far, the impact of refraction on the lightcurves of transiting exoplanets has been largely ignored, probably because most of the exoplanets amenable to in-transit spectroscopy are in orbits of only a few days. Refraction, though, may wash out some of the structure in the transmission spectrum of a planet on a long-duration orbit [13].

García Muñoz et al. [7] have explored this issue for the specific case of the Earth-Sun system, both being observed from a remote distance. For a sunlight ray grazing the Earth's surface, the bending angle is about $1^{\circ}$, which is about four times the Sun's apparent radius from the Earth. Since the bending angle is proportional to the atmospheric background densitiy, it is concluded that only altitudes above about $13 \mathrm{~km}$ (which means a background density of $1 / 4$ the sea-level value) contribute to the formation of the solar image at the planet's terminator during mid-transit. Rays crossing through lower altitudes are refracted away from the observer. Since refraction prevents the access to the near-surface atmosphere, the contrast of molecular features becomes fainter than in the non-refractive case. For transit phases other than mid-transit, other configurations occur but, as shown in Ref. [7], that does not improve the overall contrast of the spectroscopic features.

Being largely a geometrical effect, the actual impact of refraction depends on the planet's orbital distance and the stellar size, as well as on the composition of the planet's atmosphere. Thus, refraction should be considered in a case-by-case basis when evaluating the detectability of spectroscopic features for planets in the habitable zones around their host stars.

\section{THE VENUS TRANSITS OF 2004 AND 2012}

The theory of lunar eclipses is essentially identical to the refractive theory of planetary transits $[9,10]$. The Venus transits of 2004 and 2012 have provided excellent opportunities for testing the latter. In the 18th century, the realization that the Sun forms its refractive image at the Venus terminator during the transit ingress/egress meant the first evidence for a Venus atmosphere. CCD images and lightcurves of the refractive halo during the 2004 transit have been published [16], and more of them for the 2012 event will certainly be published in the coming months. García Muñoz \& Mills [6] have investigated the structure of Venus' refractive halo. For most of the phases of the ingress/egress, the refractive halo is formed within the mesosphere at altitudes less than $100 \mathrm{~km}$. In limb viewing, the Venus layer of haze can be optically thick up to altitudes of $85-90 \mathrm{~km}$, which means that the halo brightness is partly dictated by the local haze opacity. The lower altitude of the clouds near the poles explains to a large extent the brightening of the refractive halo at the Venus higher latitudes.

\section{SUMMARY}

We have reviewed the potential of lunar eclipse observations in the characterization of some of the global optical properties of the Earth's atmosphere. Investigations of this type are relevant for the accurate prediction of both continuum and molecular signatures in the transmission spectra of transiting Earth-like planets. In this respect, we emphasize the importance of radiative transfer modeling in the interpretation of the lunar eclipse observations and, subsequently, in the extrapolation to the exoplanetary case. Finally, we note that refraction may mean an additional difficulty in the characterization of the atmospheres of transiting exoplanets on long duration orbits.

The author acknowledges comments from M. R. Zapatero Osorio, R. Barrena, E. L. Martín, P. MontañésRodríguez \& E. Pallé during the elaboration of some of the work presented herein. The author expresses his 
gratitude to the organizers of the Hot Planets and Cool Stars - RoPACS Conference for their invitation to deliver an oral presentation.

\section{References}

[1] J. J. Bauman, P. B. Russell, M. A. Geller \& P. Hamill, JGR Atmospheres 108, no. D13, 4383, doi:10.1029/2002JD002993 (2003)

[2] M. Fromm, D. T. Lindsey, R. Servranckx, G. Yue, T. Trickl, R. Sica, P. Doucet \& S. GodinBeekmann, BAMS September, 1193 (2010)

[3] A. García Muñoz \& E. Pallé, JQSRT 112, 1609 (2011)

[4] A. García Muñoz, E. Pallé, M. R. Zapatero Osorio \& E. L. Martín, GRL 38, L14805 (2011)

[5] A. García Muñoz \& K. Bramstedt, JQSRT 113, 1566 (2012)

[6] A. García Muñoz \& F. P. Mills, A\&A 547, A22 (2012)

[7] A. García Muñoz, M. R. Zapatero Osorio, R. Barrena, P. Montañés-Rodríguez, E. L. Martín \& E. Pallé, ApJ 755, 103 (2012)

[8] R. Keen, Science 222, 1011 (1983)

[9] F. Link, in Physics and Astronomy of the Moon, ed. Z. Kopal (New York: Academic), 161 (1962)

[10] F. Link, Eclipse Phenomena in Astronomy (Berlin: Springer), 205 (1969)

[11] S. Matsushima, J. R. Zink \& J. E. Hansen, AJ 71, 103 (1966)

[12] E. Pallé, M. R. Zapatero Osorio, R. Barrena, P. Montañés-Rodríguez \& E. L. Martín, Nature 459, 814 (2009)

[13] E. Pallé, M. R. Zapatero Osorio \& García Muñoz, ApJ 728, doi: 10.1088/0004-637X/728/1/19 (2011)

[14] J. M. Saari \& R. W. Shorthill, Nature 205, 964 (1965)

[15] E. M. Shoemaker, R. M. Batson, H. E. Holt, E. C. Morris, J. J. Rennilson \& E. A. Whitaker, JGR 73, 3989 (1968)

[16] P. Tanga, T. Widemann, B. Sicardy, J. M. Pasachoff, J. Arnaud, L. Comolli, A. Rondi, S. Rondi \& P. Sütterlin, Icarus 218, 207 (2012)

[17] O. S. Ugolnikov \& I. A. Maslov, JQSRT 102, 499 (2006)

[18] O. S. Ugolnikov \& I. A. Maslov, JQSRT 109, 378 (2008)

[19] A. Vidal-Madjar, L. Arnold, D. Ehrenreich, R. Ferlet, A. Lecavelier Des Etangs, F. Bouchy, D. Segransan, I. Boisse, G. Hébrard, C. Moutou, J.-M. Désert, D. K. Sing, R. Cabanac, C. Nitschelm, X. Bonfils, X. Delfosse, M. Desort, R. F. Diaz, A. Eggenberger, T. Forveille, A.-M. Lagrange, C. Lovis, F. Pepe, C. Perrier, F. Pont, N. C. Santos \& S. Udry, A\&A 523, A57 (2010)

[20] J. Yamazaki, S. Mitsuhashi, M. Yamauchi, J. Tachino, R. Honda, M. Shirao, K. Tanimoto, H. Tanaka, N. Harajima, A. Omori, S. Yahagi, S. Kanayama, Y. Iijima \& H. Ohtake, Space Sci. Rev. 154, 21 (2010) 